\title{
Research Paper: Changes in Gait Pattern During Smartphone and Tablet Use
}

\author{
Sirinthip Pakdee ${ }^{1}$ (D), Praphatson Sengsoon ${ }^{1^{*}}$ (D) \\ 1. Department of Physical Therapy, School of Allied Health Sciences, Walailak University, Nakhon Si Thammarat Province, Thailand.
}

\begin{tabular}{l|l}
$\begin{array}{c}\text { Use your device toscan } \\
\text { and read the article online }\end{array}$ \\
2020; 18(4):475-484. http://dx.doi.org/10.32598/irj.18.4.1108.1 \\
doj:http://dx.doi.org/10.32598/irj.18.4.1108.1
\end{tabular}

(c) (i) (8)

Article info:

Received: 02 Apr 2020

Accepted: 08 Nov 2020

Available Online: 01 Dec 2020

\section{Keywords:}

Gait, Walking, Smartphone, Tablet, Smartphone, Tablet

\begin{abstract}
A B S T R ACT
Objectives: There exists no study concerning gait pattern while engaging in dual-task activities on different sizes of mobile devices. The present study aimed to compare gait patterns during normal walking, walking with smartphone use, and walking with tablet use.
\end{abstract}

Methods: Seventeen healthy female participants with an age range of 19-24 years (Mean \pm SD age: $20.29 \pm 1.61$ years, weight: $49.82 \pm 4.46 \mathrm{~kg}$, height: $156.59 \pm 4.87 \mathrm{~cm}$, body mass index: $20.47 \pm 0.84 \mathrm{~kg} / \mathrm{m} 2$, and leg length: $81.35 \pm 3.79 \mathrm{~cm}$.) participated in the present research. The study subjects were requested to walk for 10 meters under the following conditions: normal walking; walking while typing a text message with a smartphone, and walking while typing a text message with a tablet. The Vicon ${ }^{\circledR}$ Three-Dimensional (3D) motion analysis recorded the motion of reflective markers attached to the study participant's body. Gait parameters (step length, stride length, step time, stride time, step width, velocity, and cadence) were compared between the explored walking conditions.

Results: The gait parameter of normal walking, compared with walking during smartphone use as well as tablet use were significantly different in the gait pattern $(\mathrm{P}<0.05)$. Using either a smartphone or tablet during walking presented decreased step length for legs, gait velocity, and cadence. However, there was an increase in the right leg's step time and stride time. Moreover, there was no significant difference in gait patterns between walking during smartphone use and walking during tablet use $(\mathrm{P}>0.05)$.

Discussion: Changes in gait pattern during walking while typing a text message with either a smartphone or tablet may lead to greater attention and concentration required to type the message; such actions resulted in decreased gait speed and step length.

\section{* Corresponding Author:}

Praphatson Sengsoon, PhD.

Address: Department of Physical Therapy, School of Allied Health Sciences, Walailak University, Nakhon Si Thammarat Province, Thailand.

Tel: +66 (75) 672602-4

E-mail: praphatson.kl@wu.ac.th 


\section{Highlights}

- Walking patterns changed from normal during walking while using either a smartphone or tablet.

- Gait speed and step length were decreased during walking while typing a text message with a smartphone or tablet. Such outcomes resulted from greater attention and concentration required to type the message while walking.

- The screen size presented no effect on the gait pattern.

\section{Plain Language Summary}

Smartphones and tablets are used in everyday life, especially during walking that may affect gait patterns. This study compared gait patterns during normal walking, walking while typing a text message with a smartphone, and walking while typing a text message with a tablet. The study participants walked for a distance of 10 meters per condition. Gait patterns were analyzed by the Vicon ${ }^{\circledR} 3 \mathrm{D}$ motion analysis system. We found that using either smartphone or tablet during walking suggested decreased step length, decreased gait velocity, and decreased cadence, compared with normal walking; however, gait pattern was not different between walking during smartphone use and tablet use. Changes in gait pattern during walking while typing a text message with either a smartphone or tablet may lead to greater attention and concentration required to type the message. Accordingly, such actions may induce the risk of falling during walking.

\section{Introduction}

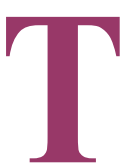

echnology plays a vital role in the Activities of Daily Living (ADL). Especially concerning communication technology, such devices as mobile phones, smartphones, tablets, desktop computers, etc., help in the convenience of communication and the easy forwarding of information. These tools are widely used for domestic and international transactions with various telecommunication networks $[1,2]$. In Thailand, using the internet and communication via smartphones and tablets are essential; they include such operations as messaging, gaming, emailing, social networking, and applying various applications [3, 4]. The features of smartphones and tablets can greatly vary, and they are easy to carry. Therefore, their application has become popular among different age groups, from youth to old ages [5]. The survey of the National Statistical Office Ministry of Information and Communication Technology in Thailand was conducted from 2011 to 2014. The relevant data suggested that using smartphones (including tablets) in the Thai population increased by $75.72 \%$. These data reflect that the future use of smartphones and tablets might be increased respecting the number of users [6].

Most individuals who use smartphones and tablets develop addictive behaviors, impacting their use in the ADLs, especially when walking on such pavements as sidewalks and roads. This issue increases the risk of falling; crashing with obstacles or other passengers, and experiencing car accidents that may cause injury and death $[3,7]$. Numerous researchers are interested in exploring the use of smartphones while walking.

Previous studies investigated using a smartphone while walking and engaging in other concurrent activities (i.e. dual-task). Accordingly, it was found that changes occurred in walking patterns. The step length, stride length, velocity, and cadence were decreased while walking during smartphone use (e.g. texting and gaming). Besides, these factors were affected by the light level and the characteristics of different walkways. Furthermore, step time, stride time, and step width were increased when using a smartphone to walk over obstacles or walk up and down ramps. Additionally, walking while using a smartphone to type text messages or read articles increased lateral deviation [3, 7-13].

Factors that resulted in changes in gait pattern while using a smartphone included postural control and balance [5, 8], and the motor system; it involves upper extremity movements where motor skills play the main role in safe walking [9]. Using smartphones or tablets while walking concerning thought processes (e.g. using increased concentration to determine location, direction, and walkway) also reduced the stability of walking and gait patterns $[8,9,14,15]$. Such a process was due to the disturbance of the sensory system, which includes the somatosensory, vestibular, and visual systems [8]. Factors other than those mentioned above that affect gait patterns include stability [3, 16] and body biomechanics [17]. Such characteristics help the body to move more efficiently while 
walking. These factors require muscle activity related to joint movement to maintain stability while walking [18].

Using smartphones and tablets could influence different gait patterns. Tablet devices are developed from smartphones with different screen widths (larger than smartphones), various sizes may differently affect cognitive processes [19]. Tablets use may require a process with less cognition and concentration than smartphones. The effect of screen size is argued to the processing time to recognize the information [19]. A previous study found the effect of the size of the smartphone screen on user's work processes concerning static posture control and dynamic posture control [2]. However, there no study explored the effect of screen size on walking. Therefore, the present research aimed to compare gait patterns during normal walking, walking while using a smartphone, and walking while using a tablet.

\section{Methods}

This was a descriptive and cross-sectional research study. The study included data collection and analysis for gait parameters under each of the three research conditions mentioned above. The study participants were 17 female students who were selected by the convenience sampling method. The number of samples in this study was calculated as per the results of a pilot study.

The inclusion criteria of the research included healthy women with the following characteristics: the age of 18-24 years [18]; normal body mass index of $18.5-22.9 \mathrm{~kg} / \mathrm{m}^{2}$ [18]; dominance in the right hand [20], and $>1$-year experience of using a smartphone or tablet for $>4$ hours per day [20].

The exclusion criteria included abnormalities or injuries to the musculoskeletal system that affect walking, such as asymmetrical leg length or scoliosis, etc. [21]; neurological disorders; using medications that affect walking and balance [22]; alcoholic beverages consumption within 24 hours before the test, and visual problems that have not been corrected or require using bifocal lenses.

Participants who met the study criteria and agreed to participate in this study received an explanation of its objectives and advantages before the test. The research participants completed questionnaires on their general information and using smartphones and tablets. The researchers conducted a physical examination according to the record form. Then, the research participants were requested to use their smartphones (iPhone6s) or tablets (iPad Air2 model) with both hands, where they held and touched the screen as desired. The tablet was held vertically with the left hand and the screen was touched with the right hand. The study participants then underwent typing training, where they must have correctly typed their nickname in English. The researchers then followed the Plug-in Gait full-body model (Figure 1). The marker was attached on participant's body as follow Table 1. The research subjects were tested by being asked to walk along a walkway for a distance of 10 meters as per the test sequence, as follows:

1. Practice walking without using a smartphone or tablet for 3 rounds, resting 1 minute per round (pause 3 minutes before starting to practice walking while using a smartphone to type a text message).

2. Practice walking while using a smartphone to type a text message. Participants must type their nickname in English with a smartphone for 3 rounds, resting 1 minute per round during practice. If they type their nickname incorrectly, they must start again to practice walking while typing their nickname with the smartphone (pause 3 minutes before starting to practice walking while using a tablet to type a text message).

3. Practice walking while using a tablet to type a text message. Participants must type their nickname in English with a tablet for 3 rounds, resting 1 minute per round during practice. If they type their nickname incorrectly, they must start again to practice walking while typing their nickname with the tablet.

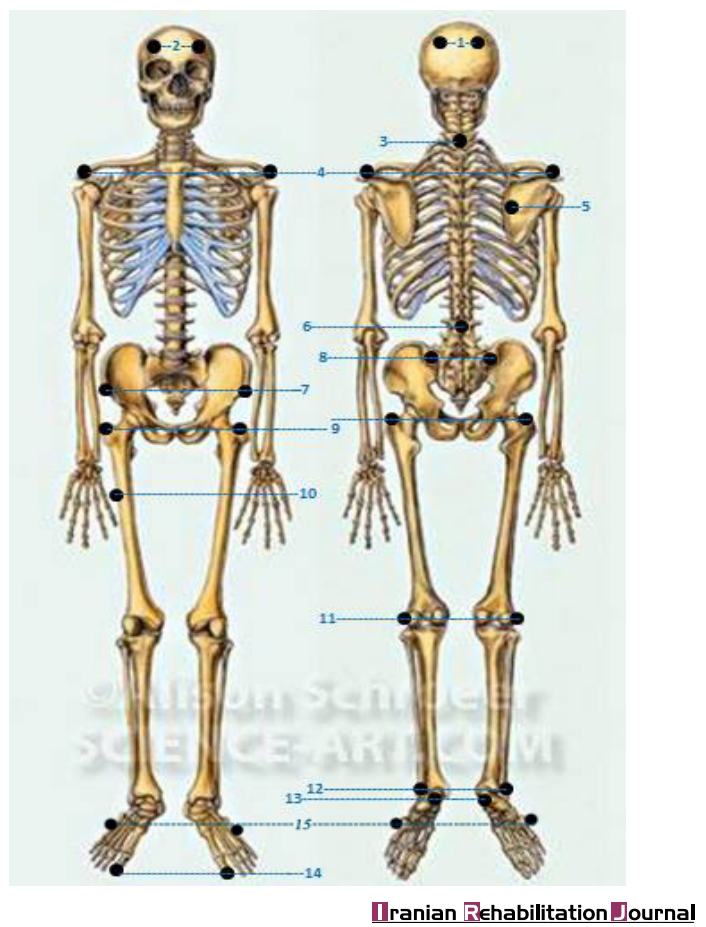

Figure 1. The Plug-in Gait-full body model 
4. Before the participants are tested, the conditions are randomized per participant (based on the draw ballot).

5. The researchers explain the process to the participants. After the practice walks, the participants start the test by walking a distance of 10 meters under the following conditions: walking without using a smartphone or tablet for 3 rounds, resting 1 minute per round, then pausing 3 minutes before starting the next walk; walking while using a smartphone for 3 rounds, resting 1 minute per round, then pausing 3 minutes before starting the next walk; and walking while using a tablet for 3 rounds, resting 1 minute per round, then pausing 3 minutes before starting the next walk.

While the participants walked on the walkway, the relevant data were recorded. Vicon ${ }^{\circledR}$ Three-Dimensional (3D) motion analysis recorded the motion of reflective markers attached to the study participant's body and processed with the Vicon Nexus program. A six-camera 3D motion-capture system at a sampling rate of $120 \mathrm{~Hz}$ was used for collecting spatial and temporal kinematic data during the walking trials. Twentysixth reflective markers were attached to specific anatomical landmarks (Figure 1). The location of the markers was detected to compute gait parameters during walking. The study variables consisted of step length (meters), stride length (meters), step time (seconds), stride time (seconds), step width (meters), velocity (meter/second), and cadence (steps/min). The researchers used the mean value of each parameter of the 3 walking cycles for analysis.

The obtained data were analyzed using SPSS at the significance level of $\mathrm{P}<0.05$. Descriptive statistics were applied to analyze the collected demographic data (mean and standard deviation), and the Kolmogorov-Smirnov test was used to test the normal distribution of the obtained data. One-way Analysis of Variance (ANOVA) was used to compare the normally-distributed data of the studied gait patterns; normal walking, walking while using a smartphone and walking while using a tablet. The Fisher's Least Significant Difference (LSD) posthoc test was used to compare the betweengroup differences.

\section{Results}

The general characteristics of the study participants are presented as follows. The research participants included 17 females with the Mean \pm SD age of $20.29 \pm 1.61$ years, Mean \pm SD weight of $49.82 \pm 4.46 \mathrm{~kg}$, Mean \pm SD height of

Table 1. Plug-in-Gait Marker placement procotol with position of the Plug-in-Gait Marker

\begin{tabular}{ccc}
\hline Marker Number & \multicolumn{1}{c}{ Position } \\
\hline 2 & (2) The back of the head & (2)The front of the head \\
3 & (1) C7 \\
4 & (2) Acromioclavicular joint \\
5 & (1) The medial boarder of Rt. scapular \\
\hline 7 & (1) L3 \\
\hline 8 & (2) ASIS \\
10 & (2) PSIS \\
11 & (2) Greater trochanter \\
12 & (1) Rt. thigh \\
13 & (2) The basis of fifth metatarsal \\
\hline 15 & (2) The lateral condyle of femur \\
\hline
\end{tabular}


Table 2. The gait parameters of normal walking and walking while using tablets and smartphones

\begin{tabular}{|c|c|c|c|c|c|}
\hline \multirow{2}{*}{ Gait Parameters } & \multirow{2}{*}{ Side } & \multicolumn{3}{|c|}{ Mean \pm SD } & \multirow{2}{*}{$\mathbf{P}$} \\
\hline & & Normal Walking & Smartphone Use & Tablet Use & \\
\hline \multirow{2}{*}{ Step length $(\mathrm{cm})$} & Right & $63.931 \pm 5.49$ & $58.002 \pm 5.71$ & $57.952 \pm 5.37$ & $0.003^{*}$ \\
\hline & Left & $62.837 \pm 5.91$ & $57.414 \pm 5.43$ & $56.858 \pm 4.76$ & $0.003^{*}$ \\
\hline \multirow{2}{*}{ Stride length $(\mathrm{cm})$} & Right & $127.173 \pm 11.28$ & $115.615 \pm 10.90$ & $114.756 \pm 10.10$ & $0.002^{*}$ \\
\hline & Left & $127.006 \pm 10.43$ & $115.472 \pm 11.29$ & $114.984 \pm 10.20$ & $0.002^{*}$ \\
\hline \multirow{2}{*}{ Step time (sec) } & Right & $0.128 \pm 0.05$ & $0.133 \pm 0.08$ & $0.133 \pm 0.01$ & $0.037^{*}$ \\
\hline & Left & $0.127 \pm 0.05$ & $0.131 \pm 0.08$ & $0.132 \pm 0.01$ & 0.187 \\
\hline \multirow{2}{*}{ Stride time (sec) } & Right & $0.253 \pm 0.01$ & $0.264 \pm 0.02$ & $0.265 \pm 0.02$ & $0.025^{*}$ \\
\hline & Left & $0.254 \pm 0.12$ & $0.265 \pm 0.16$ & $0.265 \pm 0.01$ & $0.041^{*}$ \\
\hline \multirow{2}{*}{ Step width $(\mathrm{cm})$} & Right & $8.964 \pm 1.78$ & $9.170 \pm 2.23$ & $8.499 \pm 1.87$ & 0.599 \\
\hline & Left & $7.455 \pm 2.12$ & $7.986 \pm 2.41$ & $8.317 \pm 2.18$ & 0.532 \\
\hline \multicolumn{2}{|c|}{ Velocity (meter/sec) } & $1.288 \pm 0.16$ & $1.108 \pm 0.15$ & $1.088 \pm 0.16$ & $0.001^{* *}$ \\
\hline \multicolumn{2}{|c|}{ Cadence (steps/min) } & $127.61 \pm 9.12$ & $118.60 \pm 6.98$ & $121.20 \pm 9.02$ & $0.009^{*}$ \\
\hline
\end{tabular}

Statistical analyses were performed using the one-way ANOVA $\left({ }^{*} \mathrm{P}<0.05,{ }^{* *} \mathrm{P} \leq 0.001\right)$.

Iranian Rehabilitation Journa

$156.59 \pm 4.87 \mathrm{~cm}$, Mean \pm SD body mass index of $20.47 \pm 0.84$ $\mathrm{kg} / \mathrm{m}^{2}$, and Mean \pm SD leg length of $81.35 \pm 3.79 \mathrm{~cm}$. All research participants used Apple iOS devices with a Mean \pm SD daily usage of $6.29 \pm 1.86$ hours and the results of comparison the gait parameters of normal walking and walking while using tablets and smartphones were showed in Table 2.

Comparing the differences between normal walking and walking while using a smartphone to type a text message revealed a significant difference $(\mathrm{P}<0.05)$ in step length, stride length, step time (right leg), stride time, velocity, and cadence.

Comparing the differences between normal walking and walking while using a tablet to type a message indicated a significant difference $(\mathrm{P}<0.05)$ in step length, stride length, step time (right leg), stride time, and cadence. Besides, velocity presented a significant difference in this regard $(\mathrm{P}<0.001)$. Comparing the differences between walking while using a smartphone to type a text message and walking while using a tablet to type a text message presented no significant difference in all gait parameters $(\mathrm{P}>0.05)$.

\section{Discussion}

The present study aimed to compare gait patterns during normal walking, walking while using a smartphone, and walking while using a tablet. We hypothesized that each gait pattern will be different. This is because typing while walking requires greater attention and decreases arm swing. Therefore, using smartphones or tablets while walking could change gait pattern. Besides, different screen sizes of smartphones and tablets may also affect gait pattern.

Comparing the gait pattern between normal walking and walking while using smartphones or tablets revealed a significant difference in the step length for the left and right steps. The step length was shorter while typing a text message on smartphones and tablets, i.e. consistent with the study by Licence et al. [3]; they found that the step length was reduced while using a smartphone to type a text message. Comparing the stride length in different assessment conditions demonstrated a significant difference in the stride length for both legs. Furthermore, stride length was shorter while typing a text message on smartphones and tablets. Previous studies that tested the effects of walking while typing a text message on smartphones found that stride length was also reduced [7-9]. Comparing the step time indicated that the right step time was significantly different; the right step time was increased. The obtained data concerning walking while typing a text message on smartphones and tablets were also consistent with those of Cha et al. [8]; they determined that walking across obstructions while typing a text message on smartphones increased the step time. This effect was 
Table 3. Comparing gait parameters between normal walking and walking while using a smartphone, normal walking and walking while using a tablet, and walking while using a smartphone and walking while using a tablet

\begin{tabular}{|c|c|c|c|}
\hline Gait Parameters & Side & Conditions & $\mathbf{P}$ \\
\hline \multirow{7}{*}{ Step length (cm) } & & Normal vs. Smartphone & $0.003^{*}$ \\
\hline & Right & Normal vs. Tablet & $0.003^{*}$ \\
\hline & & Smartphone vs. Tablet & 0.979 \\
\hline & & & $0005^{*}$ \\
\hline & & Normal vs. Smartphone & $0.005^{*}$ \\
\hline & Left & Normal vs. Tablet & $0.002^{*}$ \\
\hline & & Smartphone vs. Tablet & 0.765 \\
\hline \multirow{6}{*}{ Stride length $(\mathrm{cm})$} & & Normal vs. Smartphone & $0.003^{*}$ \\
\hline & Right & Normal vs. Tablet & $0.002^{*}$ \\
\hline & & Smartphone vs. Tablet & 0.817 \\
\hline & & Normal vs. Smartphone & $0.003^{*}$ \\
\hline & Left & Normal vs. Tablet & $0.002^{*}$ \\
\hline & & Smartphone vs. Tablet & 0.894 \\
\hline \multirow{7}{*}{ Step time (sec) } & & Normal vs. Smartphone & $0.022^{*}$ \\
\hline & Right & Normal vs. Tablet & $0.032^{*}$ \\
\hline & & Smartphone vs. Tablet & 0.871 \\
\hline & & & \\
\hline & & Normal vs. Smartphone & 0.152 \\
\hline & Left & Normal vs. Tablet & 0.089 \\
\hline & & Smartphone vs. Tablet & 0.783 \\
\hline \multirow{6}{*}{ Stride time (sec) } & & Normal vs. Smartphone & $0.028^{*}$ \\
\hline & Right & Normal vs. Tablet & $0.013^{*}$ \\
\hline & & Smartphone vs. Tablet & 0.748 \\
\hline & & Normal vs. Smartphone & $0.026^{*}$ \\
\hline & Left & Normal vs. Tablet & $0.031^{*}$ \\
\hline & & Smartphone vs. Tablet & 0.936 \\
\hline \multirow{6}{*}{ Step width $(\mathrm{cm})$} & & Normal vs. Smartphone & 0.761 \\
\hline & Right & Normal vs. Tablet & 0.495 \\
\hline & & Smartphone vs. Tablet & 0.325 \\
\hline & & Normal vs. Smartphone & 0.493 \\
\hline & Left & Normal vs. Tablet & 0.268 \\
\hline & & Smartphone vs. Tablet & 0.669 \\
\hline
\end{tabular}




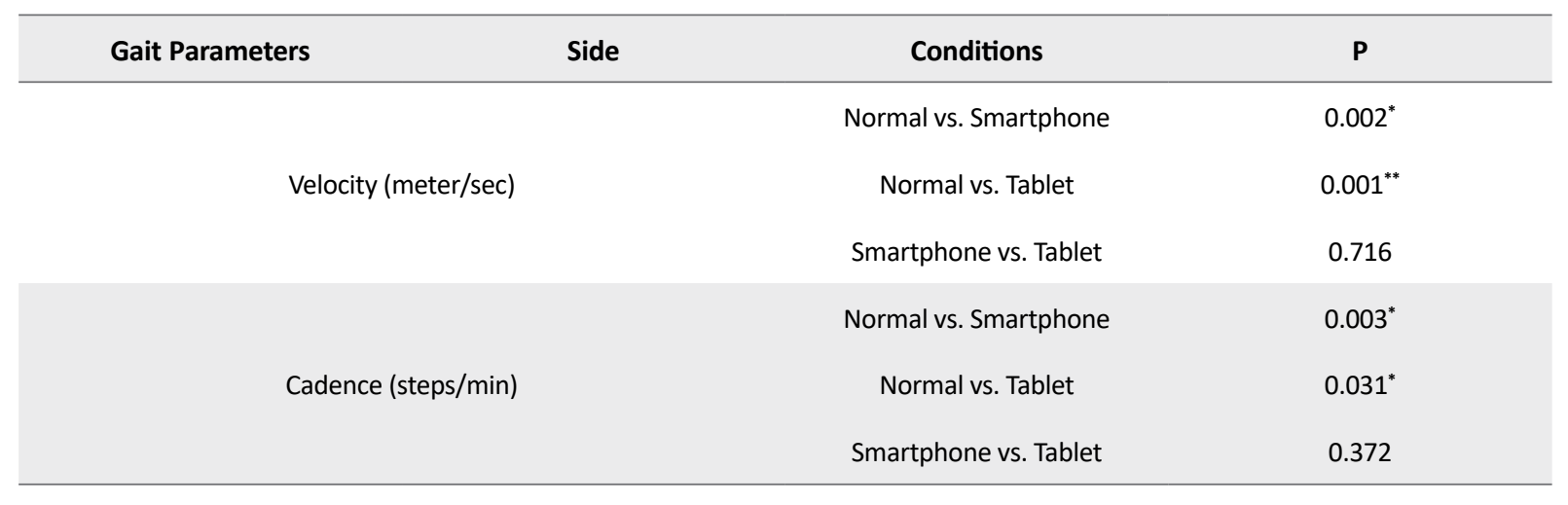

Statistical analyses were performed using posthoc test $\left({ }^{*} \mathrm{P}<0.05,{ }^{* *} \mathrm{P} \leq 0.001\right)$.

Iranian Rehabilitation Journa

related to the stability of walking. This is because walking while typing required greater concentrate and focus, causing the intention of walking to decrease [9]. The anticipatory adjustment was slower [23]; therefore, making the time spent on one step longer resulting in reduced gait stability [8]. However, the left step time was not statistically significant in this regard; the duration of one step of the left leg tended to increase when walking while typing a text message on smartphones and tablets. For the stride time, it was found that the left stride time and right stride time were significantly different. The obtained data suggested that the time required for walking equaled one step more when walking while typing a text message on smartphones and tablets. Perlmutter et al. [7] examined walking while using a smartphone to type text messages and concluded that the stride time increased. Additionally, comparing the step width between the two heels revealed no significant difference in this area. However, the left step width tended to increase when walking while using either a smartphone or tablet. According to Kao et al. [16], there was a significant difference in the step width between normal walking and walking while typing a text message on a smartphone; the step width increased for walking while typing [24]. This could be because the eyes were used for typing while walking, resulting in reduced gait stability [8, 9]. The increase in the left step width was generated to the extent of the basis of support and keep balance when moving; the center of gravity of the body shifted in inner and outer directions. Moreover, velocity was significantly different in this respect. Other studies conducted on walking while using a smartphone also found that velocity decreased $[3,11$, 13], i.e. related to a greater need for concentration or attention while using a smartphone to type a text message. There was also a significant difference in cadence. In other words, cadence decreased when walking while typing a text message on smartphones and tablets. A previous study explored walking while typing a text message on a smartphone and reported a decrease in cadence $[7,13]$.
The present study results indicated a difference in gait pattern when using smartphones or tablets. This is because using such devices while walking is a dual-task activity. A great body of literature investigated walking and performing a secondary activity; the relevant data indicated that the ability to walk decreased while performing a secondary activity. Previous studies have also explained that manual work (e.g. the physical demand of carrying a smartphone or tablet) caused reduced arm swing. Such alternations resulted in a decrease in the ability to maintain balance while walking. Therefore, using smartphones or tablets changes walking performance; decreased walking speed and increased detours may result in dangerous road crossings and unawareness of the immediate surroundings. This is because further concentration is required to look at the screen in such situations. These behaviors may eventually lead to accidents [25].

Comparing gait patterns between walking while using smartphones and tablets indicated no significant difference concerning various walking parameters. Besides, the difference in screen size did not affect cadence.

We studied the differences in gait patterns between normal walking and walking while using smartphones and tablets, where activity performed on the device involved typing a text message only. The present study overlooked including various practical uses of smartphones and tablets, such as reading text messages, playing games, talking on the phone, and so on.

The current study results can be applied to suggest the risks of walking while using smartphones and tablets. The obtained data are also applicable for preventing the dangers of such an activity, which may cause injury through tripping, colliding with obstacles, and so on. The study participants' attention seemed to be divided between the two tasks (maintaining the walking and texting on a smartphone or tablet), at the cost of affected walking ability. With increasing time being spent texting on smartphones or tablets (because of the demands of professionally-relat- 
ed tasks), the assessment of its effect on the general population is of importance in the field of public health. Due to its consequences of such walking, recommendations should be made for smartphone use. This should regard the extent of time spent on texting, the necessity of breaks, and compensatory mechanisms related to stability.

A study of a multi-task walking pattern is interesting, i.e. characterized by walking while performing various tasks, including cognitive tasks, coordination tasks, or visual tasks. Future studies should consider how such a variety of tasks can affect gait pattern. Moreover, the screen size of devices is various due to the development of technology.

\section{Conclusion}

Using smartphones and tablets adversely impact gait parameters. Walking while using smartphones and tablets decreased the step length, stride length, velocity, and cadence in the study participants. Furthermore, the right step time and stride time were increased in the study subjects. However, there was no significant difference in the left step time and step width among the study participants. There was also no significant difference between smartphone usage and tablet usage in all explored variables.

\section{Ethical Considerations}

\section{Compliance with ethical guidelines}

The study was approved by the Research Ethics Committee for research in humans, Walailak University (No. 15/090) which was performed with Declaration of Helsinki. All participants signed written informed consent forms before the commencement of the study.

\section{Funding}

This study was granted by the Department of Physical Therapy, School of Allied Health Sciences, Walailak University, Nakhon Si Thammarat Province, Thailand.

\section{Authors' contributions}

Conceptualization, writing - original draft, funding, acquisition funding, acquisition, resources: Praphatson Sengsoon; Methodology, investigation, supervision: Praphatson Sengsoon and Sirinthip Pakdee.

\section{Conflict of interest}

There is no conflicts of interest to be declared.

\section{References}

[1] Alhazmi AA, Alzahrani SH, Baig M, Salawati EM. Prevalence and factors associated with smartphone addiction among medical students at King Abdulaziz University, Jeddah. Pakistan Journal of Medical Sciences. 2018; 34(4):984-8. [DOI:10.12669/pjms.344.15294] [PMID] [PMCID]

[2] Yoon $\mathrm{CH}$. The effects of different display sizes of smart phones to the task performance. Journal of the Korea Academia-Industrial Cooperation Society. 2016; 17(4):209-14. [DOI:10.5762/KAIS.2016.17.4.209]

[3] Licence S, Smith R, McGuigan MP, Earnest CP. Gait pattern alterations during walking, texting and walking and texting during cognitively distractive tasks while negotiating common pedestrian obstacles. PloS One. 2015; 10(7):e0133281. [DOI:10.1371/journal.pone.0133281] [PMID] [PMCID]

[4] Suwannahong R. Thai students learning through smartphones. International Journal of Applied Computer Technology and Information Systems. 2018; 2 .

[5] Siu KC, Chou LS, Mayr U, van Donkelaar P, Woollacott $\mathrm{MH}$. Attentional mechanisms contributing to balance constraints during gait: The effects of balance impairments. Brain Research. 2009; 1248:59-67. [DOI:10.1016/j.brainres.2008.10.078] [PMID] [PMCID]

[6] National Statistical Office Ministry of Information and Communication Technology in Thailand. Survey the use of household information and communication technology equipment. Thailand: National Statistical Office Ministry of Information and Communication Technology; 2014.

[7] Perlmutter S, Cades DM, Heller MF, Giachetti R, Arndt SR, Sala JB. Effects of mobile technology use on walking. Proceedings of the Human Factors and Ergonomics Society Annual Meeting. 2014; 58(1):2166-70. [DOI:10.1177/1541931214581455]

[8] Cha J, Kim H, Park J, Song C. Effects of mobile texting and gaming on gait with obstructions under different illumination levels. Physical Therapy Rehabilitation Science. 2015; 4(1):32-7. [DOI:10.14474/ptrs.2015.4.1.32]

[9] Jeon S, Kim C, Song S, Lee G. Changes in gait pattern during multitask using smartphones. Work. 2015; 53(2):241-7. [DOI:10.3233/WOR-152115] [PMID]

[10] Kim H, Park J, Cha J, Song C-H. Influence of mobile phone texting on gait parameters during ramp ascent and descent. Physical Therapy Rehabilitation Science. 2014; 3(1):43-8. [DOI:10.14474/ptrs.2014.3.1.43]

[11] Lamberg EM, Muratori LM. Cell phones change the way we walk. Gait \& Posture. 2012; 35(4):688-90. [DOI:10.1016/j. gaitpost.2011.12.005]] [PMID]

[12] Schabrun SM, van den Hoorn W, Moorcroft A, Greenland C, Hodges PW. Texting and walking: Strategies for postural control and implications for safety. PloS One. 2014; 9(1):e84312. [DOI:10.1371/journal.pone.0084312] [PMID] [PMCID]

[13] Strubhar AJ, Peterson ML, Aschwege J, Ganske J, Kelley J, Schulte $\mathrm{H}$. The effect of text messaging on reactive balance and the temporal and spatial characteristics of gait. Gait \& Posture. 2015; 42(4):580-3. [DOI:10.1016/j.gaitpost.2015.09.007] [PMID] 
[14] Armieri A, Holmes JD, Spaulding SJ, Jenkins ME, Johnson AM. Dual task performance in a healthy young adult population: Results from a symmetric manipulation of task complexity and articulation. Gait \& Posture. 2009; 29(2):3468. [DOI:10.1016/j.gaitpost.2008.09.004] [PMID]

[15] Yogev-Seligmann G, Rotem-Galili Y, Mirelman A, Dickstein R, Giladi N, Hausdorff JM. How does explicit prioritization alter walking during dual-task performance? Effects of age and sex on gait speed and variability. Physical Therapy. 2010; 90(2):177-86. [DOI:10.2522/ptj.20090043] [PMID] [PMCID]

[16] Kao PC, Higginson CI, Seymour K, Kamerdze M, Higginson JS. Walking stability during cell phone use in healthy adults. Gait \& Posture. 2015; 41(4):947-53. [DOI:10.1016/j.gaitpost.2015.03.347] [PMID] [PMCID]

[17] LeVeau BF. Biomechanics of human motion: Basics and beyond for the health professions. Journal of Sports Science \& Medicine. 2010; 9:676. https://www.jssm.org/jssm-09-676. xml\%3EFulltext

[18] Lee CH, Jeon YG. Biomechanical analysis of balance and gait posture by obesity level in adolescents. International Journal of BioScience and Bio-Technology. 2014; 6(3):117-22. [DOI:10.14257/ ijbsbt.2014.6.3.14]

[19] Pórarinsdóttir JÁ. The impact of screen-use on cognitive function: A pilot study [PhD. dissertation]. Iceland: Reykjavík University; 2015. https://skemman.is/handle/1946/22566

[20] Kim JS, Lee SG, Park SK, Lee SM, Kim BK, Choi JH, et al. Comparison of grip and pinch strength between dominant and nondominant hand according to type of handedness of female college students. Journal of International Academy of Physical Therapy Research. 2011; 2(1):201-6. [DOI:10.5854/JIAPTR.2011.2.1.201]

[21] Haber CK, Sacco M. Scoliosis: Lower limb asymmetries during the gait cycle. Archives of Physiotherapy. 2015; 5:4. [DOI:10.1186/ s40945-015-0001-1] [PMID] [PMCID]

[22] George C, Verghese J. Polypharmacy and gait performance in community-dwelling older adults. Journal of the American Geriatrics Society. 2017; 65(9):2082-7. [DOI:10.1111/jgs.14957] [PMID] [PMCID]

[23] Huxham FE, Goldie PA, Patla AE. Theoretical considerations in balance assessment. The Australian Journal of Physiotherapy. 2001; 47(2):89-100. [DOI:10.1016/S0004-9514(14)60300-7]

[24] Demura S, Uchiyama M. Influence of cell phone email use on characteristics of gait. European Journal of Sport Science. 2009; 9(5):303-9. [DOI:10.1080/17461390902853069]

[25] Beurskens R, Bock O. Does the walking task matter? Influence of different walking conditions on dual-task performances in young and older persons. Human Movement Science. 2013; 32(6):1456-66. [DOI:10.1016/j.humov.2013.07.013] [PMID] 
This Page Intentionally Left Blank 